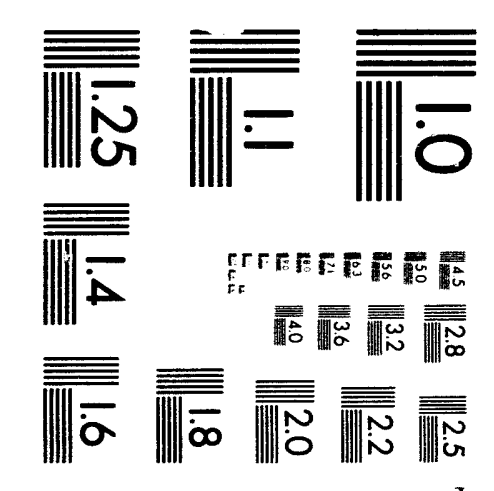



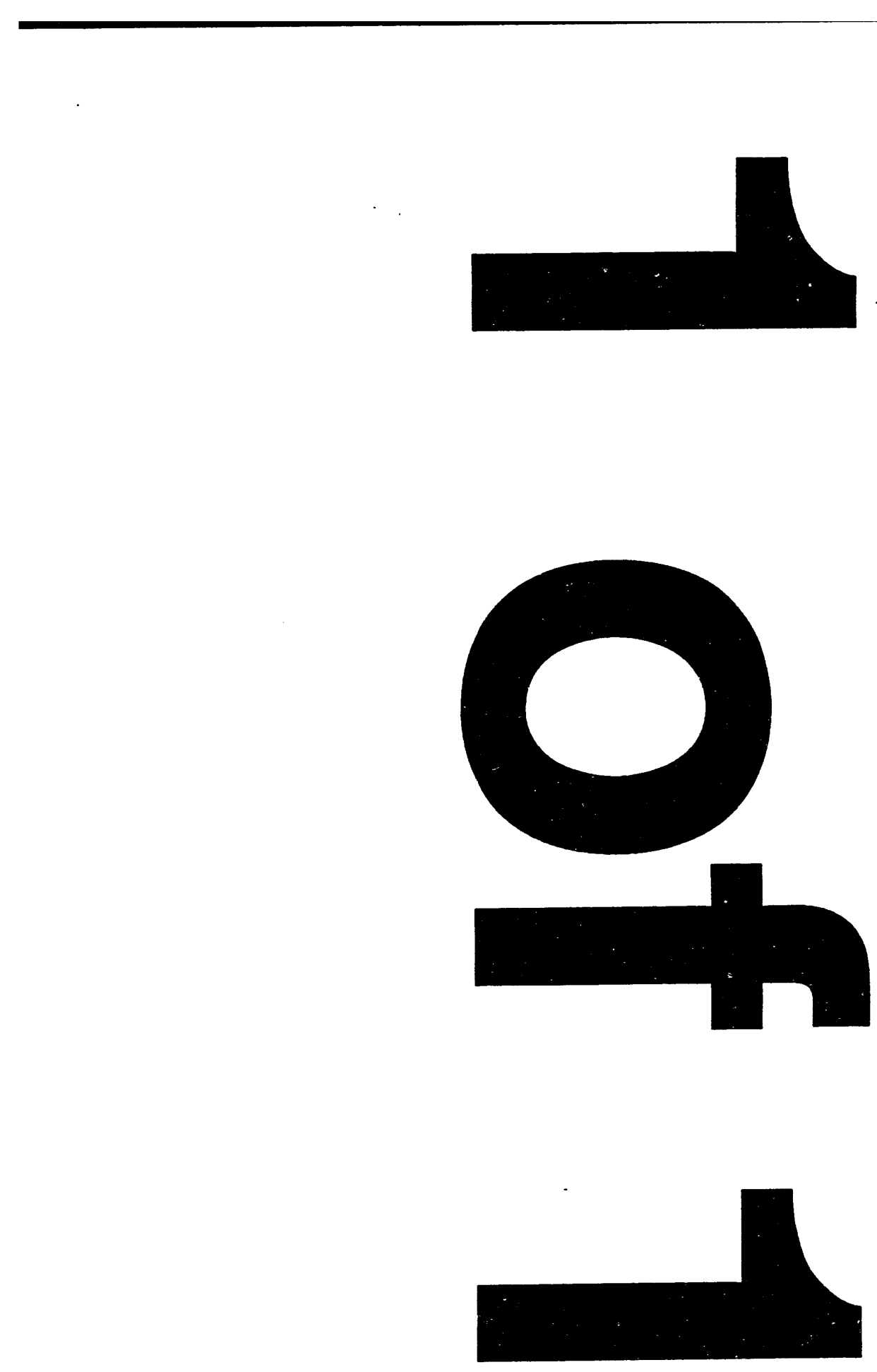


\title{
Modeling the Degradation of Nuclear Components*
}

\author{
D. Stock, ${ }^{1}$ W. Vesely, ${ }^{2}$ and P. Samanta ${ }^{1}$ \\ ${ }^{1}$ P.O. Box 5000, Brookhaven National Laboratory, Upton, NY 11973-5000 \\ ${ }^{2}$ Science Applications International Corp., 655 Metro Place S, Suite 745, Dublin, OH 43017
}

\section{Abstract}

This paper describes component level reliability models that use information on degradation to predict component reliability, and which have been used to evaluate different maintenance and testing policies. The models are based on continuous time Markov processes, and are a generalization of reliability models currently used in Probabilistic Risk Assessment. An explanation of the models, the model parameters, and an example of how these models can be used to evaluate maintenance policies are discussed.

\section{INTRODUCTION}

In order to assure the continued safe operation of aging nuclear power plants, the Nuclear Regulatory Commission is sponsoring research on the effects of aging on plant reliability. In support of this effort, Brookhaven National Laboratory (BNL) has developed component level reliability models that explicitly model degradation processes. These models have been used to plan maintenance strategies that mitigate the deleterious effects of aging.

Currently, PRA methodology is based upon reliability models that consider components to exist in only two states: operating and failed. The approach presented here identifies the degraded condition of a component as a state separate and apart from the operating and failed states. Since aging is often manifested as an increased probability of degradation, and degradation is a common path to failure, these models incorporate valuable information that is normally ignored. In addition to the degraded state, a maintenance state has been included to allow the effects of maintenance to be evaluated.

The remainder of this paper is structured as follows. Section II describes continuous time Markov Models and how these models can be used to determine the reliability parameters of components. Section III contains an example of how the model parameters can be manipulated to allow the evaluation of different maintenance and test policies. The final section provides suggestions for further reading.

\section{MARKOV MODELS}

Markov models describe the progression of an object through a series of discrete states. The class of models used here, continuous time Markov models, assume that the amount

*This work was performed under the auspices of the U.S. Nuclear Regulatory Commission. of time spent in a particular state is random, and exponentially distributed [1]. By using a Markov representation of component failure, degradation and maintenance, these events are being treated as random occurrences. While maintenance is not usually a random event, this approximation is close enough to provide useful results.

Three aspects of the Markov process are responsible for the bulk of the notation used here: time, rate of transition, and rrobability. Time will be denoted by a capital $T$ when refer.ing to the time spent in a state, and denoted with a small $t$ when used as an index. Thus, $T_{i}$ refers to the amount of time spent in state $i$, and $T_{i j}$ refers to the amount of time required to move from state $i$ to state $j$. A lower case $t$ will be used in the usual way to index functions of time. The reciprocal of $T_{i}$, which is the rate of transition out of state $i$ to any other state, will be denoted by $\lambda_{i}$. The transition rate from state $i$ to a particular state $j$ will be denoted by $\lambda_{i j}$. Lastly, the unconditional probability of being in a particular state $i$ will be denoted by $\mathrm{p}_{\mathrm{i}}$, and the conditional probability of transition from state $i$ to state $j$, given that the component is in state $i$, will be denoted by $p_{i j}$.

Current models used in PRAs assume components exist in only two states, a failed state and an operating state; intermediate degraded states are not explicitly modeled. The models used here contain two additional states, a degraded state and a maintenance state. Although the definition of degradation is somewhat arbitrary, as used here the word implies that the performance of a component has been compromised, but that the component is still operating within minimally acceptable design criteria. Precise definitions of degradation will vary with the type of component. For some components it maybe desirable to define multiple degraded states in order to reflect different types of degradation. The machinery discussed here can easily be adapted to multiple degraded states. The maintenance state is defined here to be routine maintenance or maintenance performed to ameliorate degradation. Repair, on the other hand, is defined to be actions take to restore a component to the operating condition after failure. Hence, the time required for repair has been incorporated into the failed state, and is not explicitly modeled. In the notation an $o$ will denote the operational state, $d$ will denote the degraded state, $m$ will represent the maintenance state, and $f$ will represent the failed state.

The structure of a Markov model is represented by a transition matrix. This matrix shows the allowable state-tostate transitions, and the rate of these transitions. The transition matrix for the four state model considered here is:
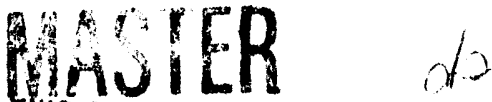
Next State

\begin{tabular}{|c|c|c|c|c|c|}
\hline \multirow[b]{3}{*}{$\begin{array}{l}\text { Current } \\
\text { State }\end{array}$} & & 0 & d & $\mathbf{m}$ & f \\
\hline & o & 0 & $\lambda_{\mathrm{od}}$ & $\lambda_{\alpha m}$ & $\lambda_{d r}$ \\
\hline & d & 0 & 0 & $\lambda_{d m}$ & $\lambda_{d r}$ \\
\hline & & $\lambda_{\mathrm{mo}}$ & $\lambda_{m d}$ & 0 & $\lambda_{\mathrm{mf}}$ \\
\hline & f & $\lambda_{10}$ & $\lambda_{f d}$ & 0 & 0 \\
\hline
\end{tabular}

It can be seen from the matrix that no transitions are allowed from degradation to operation, or from failure to maintenance. Transition from the degraded state to the operational state must happen through a sequence of additional states, such as through maintenance. There is no transition from failure to maintenance, since, as mentioned previously, the time required to restore a component from failure has been modeled as part of the failure state.

The probability that a component is in the operational, degraded, maintenance or failed state $\left(p_{o}, p_{d}, p_{m}\right.$, or $\left.p_{f}\right)$ can be determined from the steady state solution to the balance equations. If $p^{\prime}, p_{d}^{\prime}, p_{m}^{\prime}$, and $p^{\prime}{ }_{f}$ are the derivatives of the probabilities with respect to time, then the matrix form of the balance equations is:

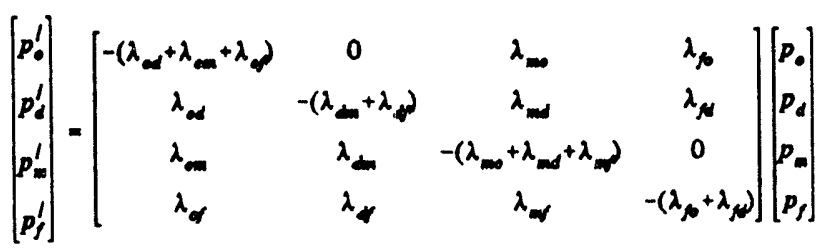

These equations imply that the rate of change in probability of a state is equal to the probability inflow to that state minus the probability outflow from that state. The steady state equation is achieved when the derivatives are all equal to zero. To solve the matrix equation, eliminate one of the four scalar equations, and add the constraint that the probabilities must sum to 1.0. This results in the following system of equations:

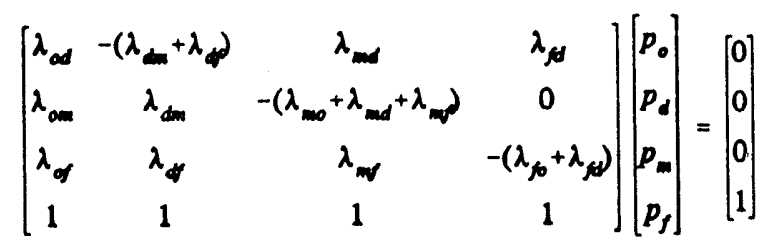

When the $\lambda_{y} s$ are known, the steady state probabilities can be found directly.
The distribution of the time to failure can be obtained by treating failure as an absorbing state, which is a state that objects can enter but not leave. This requires that $\lambda_{10}$ and $\lambda_{\text {fod }}$ both be set equal to zero. The distribution of $p_{r}(t)$ is obtained by solving the balance equations using numerical time stepping. Thus, a value of $p_{r}\left(t_{i}\right)$ is obtained at each time step, $t$, and the cumulative distribution of the probability of failure as a furction of time is obtained.

\section{EXAMPLE: COMPARISON OF TWO MAINTENANCE POLICIES}

One advantage of the models discussed here is that they can be used to compare the effectiveness of different maintenance strategies. Since resources are always limited, this approach is useful in determining how to allocate those resources. As an example of how these evaluations can be accomplished, the predicted outcomes of two maintenance policies will be compared. Under the first policy, maximal effort is expended to repair the component after failure. However, to compensate for the resources expended by the extensive repair efforts, the components are given less careful attention during maintenance. Under the second policy, maximal effort is expended on the components during maintenance. In this case, the resources expended during maintenance are compensated for by giving the components less careful attention after failure, i.e, minimal repair is performed. Both maintenance policies will be examined under surveillance testing conditions that range from continuous monitoring to a semiannual test.

First, consider the representation of the test interval in the model. If the surveillance test detects failures only, then the time spent in the degraded state will be unaffected by the tes: interval, $T_{1}$. However, the average time spent in the failex state, $T_{f}$, is equal to one half the test interval plus the average repair time, $T_{r}$. By the properties of Markov models, it is the case that:

$$
\lambda_{f 0}=p_{f_{0}} \frac{1}{\frac{T_{t}}{2}+T_{r}}=p_{f_{0}} \lambda_{f}
$$

and

$$
\lambda_{f d}=p_{f d} \frac{1}{\frac{T_{t}}{2}+T_{r}}=p_{q-f f}
$$

Here $p_{f o}$ is the probability of a failed part operating state after repair, while $p_{s a}$ is the probability of a failed part receiving improper repair, and being left in the degraded state. The parameter $p_{10}$, the probability of proper repair, is referred to as the repair efficiency. The compliment of $p_{f o s}, p_{f d}$, is referred to as the repair inefficiency. 
The efficiency of maintenance is reflected through analogous conditional probabilities. The parameters which reflect maintenance practices can be written as:

$$
\begin{aligned}
& \lambda_{m 0}=p_{m 0} \frac{1}{T_{m}}=p_{m o} \lambda_{m} \\
& \lambda_{m d}=p_{m d} \frac{1}{T_{m}}=p_{m d} \lambda_{m} \\
& \lambda_{m f}=p_{m} \frac{1}{T_{m}}=p_{m} \lambda_{m}
\end{aligned}
$$

Where $T_{m}$ is the time spent in maintenance, $p_{m o}$ is the maintenance efficiency, and the sum of $p_{m d}$ and $p_{m f}$ is the maintenance inefficiency. In this example it assumed that $p_{m f}$ is equal to zero.

A maximal expenditure of maintenance or repair effort will be represented by a down time of 72 hours, and an efficiency of 1.0. The less careful efforts will be represented by a down time of 12 hours, and an efficiency of 0.50 . Thus, under the first maintenance policy, where maximal effort is made to effect repairs after failure, the average repair time $\left(T_{\text {, }}\right.$ ) is set to 72 hours, and the repair efficiency $\left(p_{t_{0}}\right)$ to 1.0 . Since, under the first policy, less careful efforts are made during routine maintenance, the maintenance downtime $\left(T_{m}\right)$ is set to 12 hours and the maintenance efficiency $\left(p_{m o}\right)$ to 0.50 . In the second maintenance policy, the average repair time is set to 12 hours, the repair efficiency to 0.50 , the maintenance downtime to 72 hours, and the maintenance efficiency to 1.0. Under both maintenance policies, the surveillance test interval was varied from continuous monitoring ( 0 hours) to a semiannual test ( 4380 hours). The transition rates not affected by either the test interval or the maintenance policy were set to typical values.

Figure 1 shows the failure probability under both maintenance policies as a function of the surveillance test interval. The second maintenance policy, where more effort is spent in maintenance than after failure, has a much lower failure probability. Doing good maintenance prevents the degradations that lead to failure. Also, since under the second maintenance policy less time is spent doing repair upon failure, the component will spend less time in the failed state simply due to the shorter repair time. The situation is reversed when unavailability is considered. In terms of unavailability the first maintenance policy, where components receive good repairs after failure, is superior. However, the loss of component availability due to maintenance can be reduced by scheduling maintenance during plant outages.

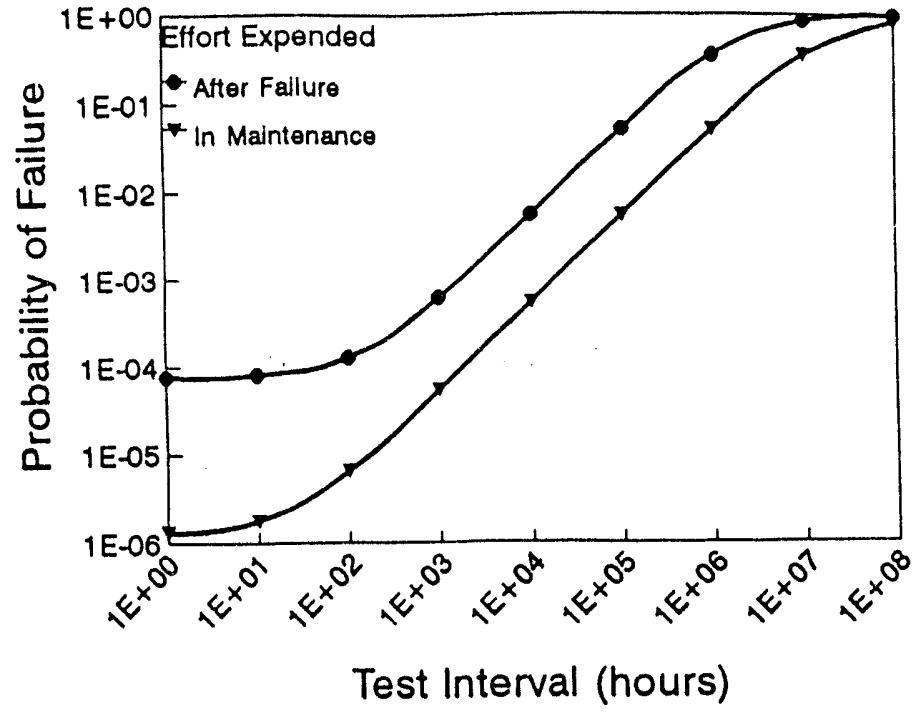

Fig. 1 Probability of failure as a function of test interval for two maintenance schedules

\section{CONCLUSIONS}

Incorporating degraded and maintenance states into component level reliability models provides a powerful tool that can be used to predict the reliability characteristics ofcomponents under a wide variety of conditions. In addition, these models can be used to evaluate different maintenance and testing scenarios. Detailed discussion of the approach outlined here can be found in NUREG/CR-5967 [5]. Earlier work on modeling degradation processes can be fourd in NUREG/CR-5612 [4], and BNL Technical Report A-3270 [2].

\section{REFERENCES}

[1] A.T. Bharucha-Reid, Elements of the Theory of Markov Processes and Their Applications, New York: McGraw Hill, 1960.

[2] F. Hsu, W.E. Vesely, E. Grove, M. Subudhi, and P.K. Samanta, "Degradation Modeling: Extensions and Applications," BNL Technical Report A-3270 6-21-91, June 1991.

[3] S.M. Ross, Introduction to Probability Models, New York: Academic Press, 1989.

[4] P.K. Samanta, W.E. Vesely, F. Hsu, and M. Subudhi, * "Degradation Modeling with Application to Aging and Maintenance Effectiveness Evaluations," NUREG/CR5612, BNL-NUREG-52252, March 1991.

[5] D.A. Stock, W.E. Vesely, and P.K. Samanta, "Development and Application of Degradation Modeling to Define Maintenance Practices, "NUREG/CR-5967, BNLNUREG-52353, July 1993. 


\section{DISCLAIMER}

This report was prepared as an account of work sponsored by an agency of the United States Government. Neither the Uxited States Government nor any agency thereof, nor any of their employees, makes any warranty, express or implied, or assumes any legal liability or responsibility for the accuracy, completeness, or usefulness of any information, apparatus, product, cr process disclosed, or represents that its use would not infringe privately owned rights. Reference herein to any specific commercial product, process, or service by trade name, trademark, manufacturer, or otherwise does not necessarily constitute or imply its endorsement, recommendation, or favoring by the United States Government or any agency thereof. The views and opinions of authors expressed herein do not necessarily state or reflect those of the United States Government or any agency thereof. 


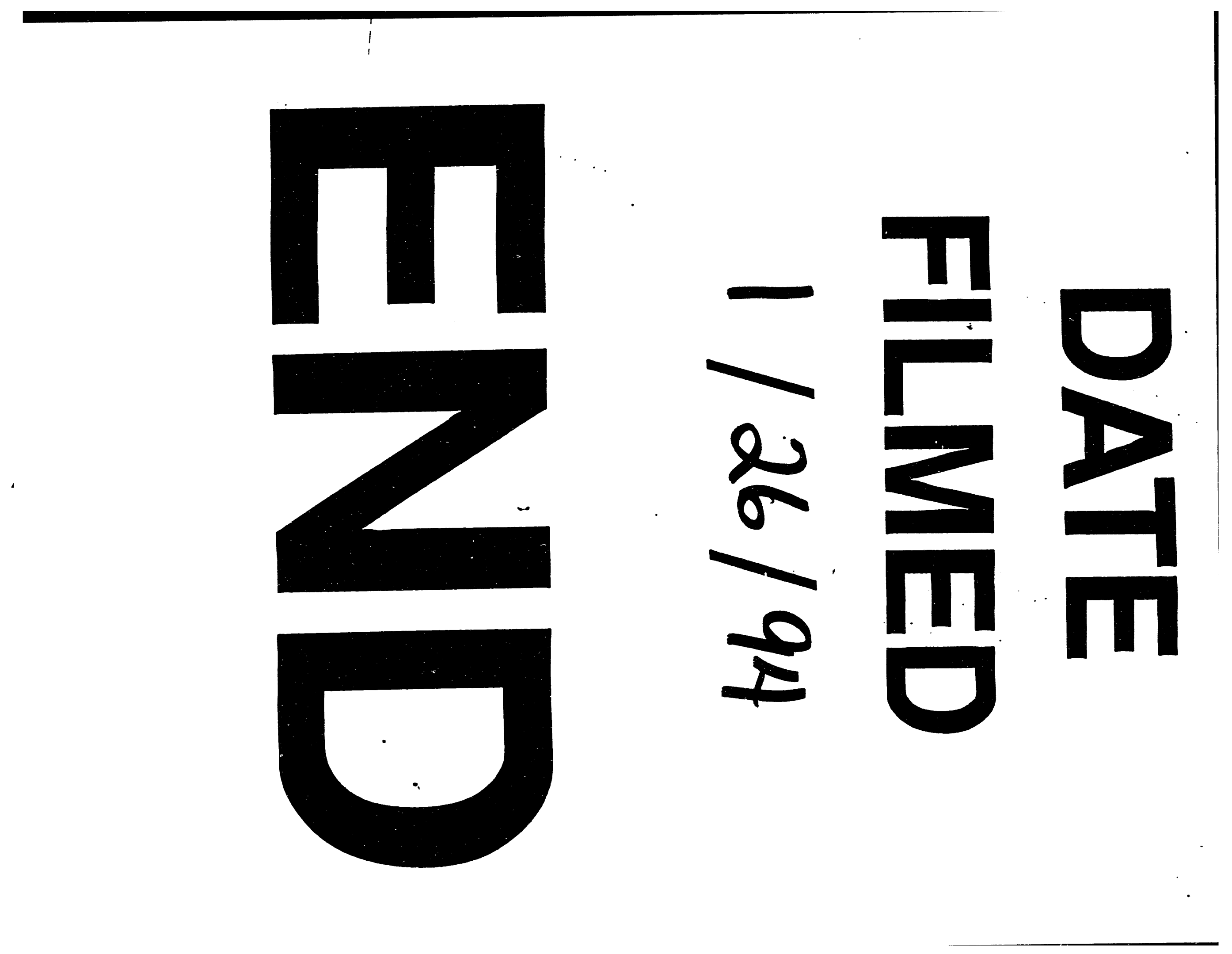


